JOURNAL OF

SYMPLECTIC GEOMETRY

Volume 1, Number 1, 47-61, 2001

\title{
Minimal annuli with and without slits
}

\author{
Tobias H. Colding and William P. Minicozzi II ${ }^{1}$
}

\section{Introduction.}

In this paper we bound the oscillation of the unit normal of minimal annuli with and without slits. Our estimates are independent of the ratio of the inner and outer radii. Hence, we recover standard removable singularity results as the inner radius goes to zero. The estimate for annuli with slits is important in proving a removable singularities theorem for minimal limit laminations.

Proposition 1.3 shows that if a minimal annulus $\Sigma$ in $\mathbf{R}^{3}$ has $\int_{\partial \Sigma}|A|<$ $\pi / 8$ and $\int_{\Sigma} K \geq-\pi$, then $\Sigma$ is a graph. Proposition 1.12 extends this to surfaces with quasi-conformal Gauss map and shows that if $\int_{\partial \Sigma}|A|$ is actually small, then $\Sigma$ is Lipschitz close to a plane. These results, as well as the rest of the results of this paper, can be easily extended to hold locally in any 3 -manifold.

In Section 3 we extend this to what we call "minimal annuli with slits". These are multi-valued minimal graphs over an annulus in the plane. A particular example is a rotation of a rescaled half-helicoid:

$$
\{\epsilon \delta /(2 \pi)(s \cos t, s \sin t, t) \mid 2 \pi / \epsilon \leq s \leq 2 \pi R /(\epsilon \delta) \text { and } 0 \leq t \leq 2 \pi\} .
$$

Since $\mathbf{n}(\epsilon \delta /(2 \pi)(s \cos t, s \sin t, t))=(\sin t,-\cos t, s) /\left(1+s^{2}\right)^{1 / 2}$ and $s \geq$ $2 \pi / \epsilon$

$$
|\mathbf{n}-(0,0,1)|<\epsilon \text { in }(0.1)
$$

independent of $\delta$ and $R$. In Theorem 3.36, we obtain a similar bound in general for minimal annuli with slits satisfying certain boundary conditions. This bound implies that there is a fixed plane which these are Lipschitz

\footnotetext{
${ }^{1}$ The first author was partially supported by NSF Grant DMS 9803253 and an Alfred P. Sloan Research Fellowship and the second author by NSF Grant DMS 9803144 and an Alfred P. Sloan Research Fellowship.
} 
close to on every scale. This consequence will be used elsewhere to prove the removability of singularities for minimal limit laminations.

The results given here should be compared with Rado's theorem (see for instance $[\mathrm{CM}]$ ) which states that a minimal surface in $\mathbf{R}^{3}$ whose boundary is a circle which is a graph over the boundary of a convex set in a plane is itself a graph (and in fact a disk).

Throughout, $\Sigma \subset \mathbf{R}^{3}$ is a compact connected oriented immersed surface; $A, \nabla_{\Sigma}, \Delta_{\Sigma}, \mathbf{n}, K$ are the second fundamental form, covariant derivative, Laplacian, unit normal, and sectional curvature. Given $x \in \mathbf{R}^{3}$ and $s>0$, $B_{s}(x)$ is the extrinsic ball of radius $s$ centered at $x$. Likewise $D_{s}(z)$ will be the disk in the plane centered at $z$ and with radius $s$. Finally, $\mathcal{B}_{s}(a)$ is the intrinsic ball in $\mathbf{S}^{2}$ of radius $s$ centered at $a \in \mathbf{S}^{2}$.

\section{Minimal annuli.}

In this section, if $a \in \mathbf{S}^{2}, a^{\perp}$ denotes $\left\{x \in \mathbf{R}^{3} \mid\langle x, a\rangle=0\right\}$. For $a, b \in \mathbf{S}^{2}$, $\operatorname{Angle}(a, b)$ is the angle between $a^{\perp}, b^{\perp}$; i.e., $\operatorname{Angle}(a, b)=\operatorname{dist}_{\mathbf{S}^{2}}(a,\{b,-b\})$.

Let $f$ be harmonic on $\Sigma^{2}$ with critical points $\left\{y_{i}\right\}$ with multiplicities $\left\{m_{i}\right\}$. Suppose that none of the $y_{i}$ 's lie on $\partial \Sigma$. The Bochner formula on $\Sigma \backslash\left\{y_{i}\right\}$ gives

$$
\Delta_{\Sigma} \log \left|\nabla_{\Sigma} f\right|^{2}=2 \frac{\left|\operatorname{Hess}_{f}\right|^{2}}{\left|\nabla_{\Sigma} f\right|^{2}}+2 K-\frac{\left.\left.\left|\nabla_{\Sigma}\right| \nabla_{\Sigma} f\right|^{2}\right|^{2}}{\left|\nabla_{\Sigma} f\right|^{4}}=2 K
$$

Here we used that since $\Delta_{\Sigma} f=0$, then $2\left|\operatorname{Hess}_{f}\right|^{2}\left|\nabla_{\Sigma} f\right|^{2}=\left.\left.\left|\nabla_{\Sigma}\right| \nabla_{\Sigma} f\right|^{2}\right|^{2}$. Hence, by Stokes' theorem

$$
\begin{aligned}
\int_{\partial \Sigma} \frac{d \log \left|\nabla_{\Sigma} f\right|^{2}}{d n} & =\int_{\Sigma \backslash\left\{y_{i}\right\}} \Delta_{\Sigma} \log \left|\nabla_{\Sigma} f\right|^{2}+4 \pi \sum_{i} m_{i} \\
& =2 \int_{\Sigma} K+4 \pi \sum_{i} m_{i}
\end{aligned}
$$

Proposition 1.3. If $\Sigma$ is connected and minimal with boundaries $\sigma_{1}$ and $\sigma_{2}, \int_{\sigma_{1} \cup \sigma_{2}}|A|<\pi / 8$, and $\int_{\Sigma} K \geq-\pi$, then $\Sigma$ is graphical.

Proof. Fix $q_{i} \in \sigma_{i}$. Since $\left|\nabla_{\Sigma} \operatorname{dist}_{\mathbf{S}^{2}}\left(\mathbf{n}\left(q_{i}\right), \mathbf{n}(\cdot)\right)\right| \leq|A|$, the assumption on $\partial \Sigma$ gives

$$
\sum_{i} \sup _{z_{i} \in \sigma_{i}} \operatorname{dist}_{\mathbf{S}^{2}}\left(\mathbf{n}\left(q_{i}\right), \mathbf{n}\left(z_{i}\right)\right) \leq \sum_{i} \int_{\sigma_{i}}|A|<\pi / 8
$$


Choose $b \in \mathbf{S}^{2}$ with $\operatorname{Angle}\left(\mathbf{n}\left(q_{i}\right), b\right) \leq \pi / 4$ for $i=1,2$. We will show that $\Sigma$ is graphical over the plane $b^{\perp}$. By the triangle inequality and (1.4), for $i=1,2$,

$$
\sup _{z_{i} \in \sigma_{i}} \operatorname{Angle}\left(b, \mathbf{n}\left(z_{i}\right)\right) \leq \pi / 4+\sup _{z_{i} \in \sigma_{i}} \operatorname{dist}_{\mathbf{S}^{2}}\left(\mathbf{n}\left(q_{i}\right), \mathbf{n}\left(z_{i}\right)\right)<3 \pi / 8 .
$$

Rotate coordinates so that $b=(0,0,1)$ and $b^{\perp}$ is the $x_{1}-x_{2}$-plane. Fix $\theta$ and set $f=x_{1} \cos \theta+x_{2} \sin \theta$. Given $x \in \Sigma$,

$$
\left|\nabla_{\Sigma} f\right|^{2}(x)=1-\langle(\cos \theta, \sin \theta, 0), \mathbf{n}(x)\rangle^{2} \geq\langle b, \mathbf{n}(x)\rangle^{2} .
$$

On $\partial \Sigma=\sigma_{1} \cup \sigma_{2},(1.5)$ and (1.6) imply that

$$
\inf _{\partial \Sigma}\left|\nabla_{\Sigma} f\right| \geq \inf _{\partial \Sigma}|\langle b, \mathbf{n}(x)\rangle|>\cos (3 \pi / 8)>1 / 3 .
$$

Since $\left|\nabla_{\Sigma}\right| \nabla_{\Sigma} f|| \leq\left|\operatorname{Hess}_{f}\right| \leq|A|,(1.7)$ gives on $\partial \Sigma$

$$
\left.\left|\nabla_{\Sigma} \log \right| \nabla_{\Sigma} f\right|^{2}|=2| \nabla_{\Sigma}\left|\nabla_{\Sigma} f\right||/| \nabla_{\Sigma} f|\leq 6| A \mid
$$

Integrating (1.8), we get

$$
\int_{\partial \Sigma}\left|\frac{d \log \left|\nabla_{\Sigma} f\right|^{2}}{d n}\right| \leq 6 \int_{\partial \Sigma}|A|<3 \pi / 4
$$

Since $\Sigma$ is minimal, $\Delta_{\Sigma} f=0$. Substituting (1.9) into (1.2),

$$
4 \pi \sum_{i} m_{i}=\int_{\partial \Sigma} \frac{d \log \left|\nabla_{\Sigma} f\right|^{2}}{d n}-2 \int_{\Sigma} K<3 \pi / 4+2 \pi<4 \pi ;
$$

hence, $f$ has no critical points. Since this is true for any $\theta, \Sigma$ is graphical over $b^{\perp}$.

We next generalize Proposition 1.3 to $\Sigma$ with $\mathbf{n}$ quasi-conformal. We will use that if $\Omega \subset \mathbf{S}^{2}$ is connected and $\operatorname{diam}_{\mathbf{S}^{2}}(\Omega)<\pi / 2$, then by the maximum principle

$$
\operatorname{diam}_{\mathbf{S}^{2}}(\Omega)=\operatorname{diam}_{\mathbf{S}^{2}}(\partial \Omega) ;
$$

here we used that $\operatorname{dist}_{\mathbf{S}^{2}}^{2}(z, \cdot)$ is convex on $\mathcal{B}_{\pi / 2}(z) \subset \mathbf{S}^{2}$. 
Proposition 1.12. If $\Sigma \subset \mathbf{R}^{3}$ is connected, $\int_{\Sigma}|K| \leq \pi,|A|^{2} \leq C|K|$, and $\partial \Sigma$ has components $\left\{\sigma_{i}\right\}_{1 \leq i \leq n}$ with

$$
\sum_{i=1}^{n} \inf _{a \in \mathbf{S}^{2}} \sup _{x \in \sigma_{i}}\left\{\operatorname{dist}_{\mathbf{S}^{2}}(\mathbf{n}(x), a)\right\}<\epsilon<\pi / 8,
$$

then $\mathbf{n}(\Sigma) \subset \mathcal{B}_{2 \epsilon}(a)$ for some $a \in \mathbf{S}^{2}$ and $\Sigma$ is the graph of $u$ over $a^{\perp}$ with $|\nabla u| \leq 4 \epsilon$.

Proof. Choose $a_{i} \in \mathbf{S}^{2}$ and $\epsilon_{i}>0$ so that $\mathbf{n}\left(\sigma_{i}\right) \subset \mathcal{B}_{\epsilon_{i}}\left(a_{i}\right) \subset \mathbf{S}^{2}$ for each $i$, $\sum_{i} \epsilon_{i}<\epsilon, \operatorname{dist}_{\mathbf{S}^{2}}\left(a_{i}, a_{j}\right) \neq \epsilon_{i}+\epsilon_{j}$, and $\operatorname{dist}_{\mathbf{S}^{2}}\left(a_{i}, a_{j}\right) \neq\left|\epsilon_{i}-\epsilon_{j}\right|$ for $i \neq j$ (i.e., the $\partial \mathcal{B}_{\epsilon_{i}}\left(a_{i}\right)$ 's are transverse). Let $\Omega_{1}, \ldots, \Omega_{m}$ be the connected components of $\cup_{i=1}^{n} \overline{\mathcal{B}_{\epsilon_{i}}}\left(a_{i}\right)$, so that

$$
\sum_{i=1}^{m} \operatorname{diam}_{\mathbf{S}^{2}}\left(\Omega_{i}\right) \leq \sum_{i=1}^{n} \operatorname{diam}_{\mathbf{S}^{2}}\left(\mathcal{B}_{\epsilon_{i}}\left(a_{i}\right)\right)<2 \epsilon<\pi / 4 .
$$

Since the $\partial \mathcal{B}_{\epsilon_{i}}\left(a_{i}\right)$ 's are transverse, each $\partial \Omega_{i}$ is a finite union of transverse circular arcs. Hence, there are (closed) disks $\Omega_{i}^{0}$ with $\Omega_{i} \subset \Omega_{i}^{0}, \partial \Omega_{i}^{0} \subset \partial \Omega_{i}$, and $\operatorname{diam}_{\mathbf{S}^{2}}\left(\Omega_{i}^{0}\right)=\operatorname{diam}_{\mathbf{S}^{2}}\left(\Omega_{i}\right)$ (using (1.11)). Since $\partial \Omega_{i}^{0} \cap \partial \Omega_{j}^{0}=\emptyset$ if $i \neq j$, a disjoint subset (after reordering) $\Omega_{1}^{0}, \ldots, \Omega_{q}^{0}$ has

$$
\cup_{i=1}^{n} \mathbf{n}\left(\sigma_{i}\right) \subset \cup_{i=1}^{m} \Omega_{i} \subset \cup_{i=1}^{q} \Omega_{i}^{0} .
$$

Since $\operatorname{diam}_{\mathbf{S}^{2}}\left(\Omega_{i}^{0}\right)=\operatorname{diam}_{\mathbf{S}^{2}}\left(\Omega_{i}\right),(1.14)$ and the volume comparison imply that

$$
\sum_{i=1}^{q} \operatorname{Area}\left(\Omega_{i}^{0}\right)<\pi \sum_{i=1}^{q} \operatorname{diam}_{\mathbf{S}^{2}}^{2}\left(\Omega_{i}^{0}\right) \leq \pi \sum_{i=1}^{n} \operatorname{diam}_{\mathbf{S}^{2}}^{2}\left(\Omega_{i}\right)<\pi(\pi / 4)^{2}<\pi .
$$

Since the $\Omega_{i}^{0}$ 's are disjoint disks,

$$
\Omega=\mathbf{S}^{2} \backslash \cup_{i=1}^{q} \Omega_{i}^{0}
$$

is a connected open set with $q$ boundary components. We show next that $q=1$. If $q>1$, then $\mathcal{H}^{1}(\mathbf{n}(\Sigma) \cap \Omega) \geq \operatorname{dist}_{\mathbf{S}^{2}}\left(\Omega_{1}^{0}, \Omega_{2}^{0}\right)>0$ since $\Sigma$ is connected and $\mathbf{n}(\Sigma) \cap \Omega_{i}^{0} \neq \emptyset$ for each $i$. Here $\mathcal{H}^{1}$ is the one-dimensional Hausdorff measure. Hence, since $\bar{\Omega} \cap \mathbf{n}(\partial \Sigma)=\emptyset$, Lemma A.1 implies that $\Omega \subset \mathbf{n}(\Sigma)$. However, together with (1.16), this would imply that

$$
3 \pi<4 \pi-\operatorname{Area}\left(\cup_{i=1}^{q} \Omega_{i}^{0}\right)=\operatorname{Area}(\Omega) \leq \operatorname{Area}(\mathbf{n}(\Sigma)) \leq \int_{\Sigma}|K| \leq \pi
$$


so that we must have $q=1$. Hence, $\mathbf{n}(\Sigma) \subset \Omega_{1}^{0}$ and the claim follows from (1.14).

\section{Holomorphic functions on annuli.}

We will now obtain $C^{0,1}$ estimates for holomorphic functions on annuli as a model for the Gauss map of a minimal annulus with a slit. The next section extends these arguments to that case.

Lemma 2.1. If $f: D_{R} \backslash D_{\delta} \rightarrow \mathbf{C}$ is holomorphic and $\int_{\partial D_{R} \cup \partial D_{\delta}}|\nabla f| \leq \epsilon$, then

$$
\min _{c \in \mathbf{C}} \max _{z}|f(z)-c| \leq \epsilon
$$

Proof. For $\delta \leq s \leq R$, set

$$
I(s)=(2 \pi s)^{-1} \int_{\partial D_{s}} f=(2 \pi)^{-1} \int_{0}^{2 \pi} f\left(s \mathrm{e}^{i \theta}\right) d \theta,
$$

and $c=I(\delta)$. Differentiating (2.3), we have

$$
\begin{aligned}
2 \pi s I^{\prime}(s) & =\int_{\partial D_{s}} \frac{\partial f}{\partial r}=-i s^{-1} \int_{\partial D_{s}} \frac{\partial f}{\partial \theta}=-i \int_{0}^{2 \pi} \frac{\partial f}{\partial \theta} d \theta \\
& =-i\left[f\left(s \mathrm{e}^{i 2 \pi}\right)-f\left(s \mathrm{e}^{0}\right)\right]=0,
\end{aligned}
$$

where we used that $\frac{\partial f}{\partial r}=-i r^{-1} \frac{\partial f}{\partial \theta}$ since $f$ is holomorphic. In particular, $I(R)=c$. Since $I(s)$ is the average of $f$ over $\partial D_{s}$, there exist $y_{1}, y_{2} \in \partial D_{R}$ with

$$
c=\operatorname{Re}\left(f\left(y_{1}\right)\right)+i \operatorname{Im}\left(f\left(y_{2}\right)\right) .
$$

Combining (2.5) with $\int_{\partial D_{R} \cup \partial D_{\delta}}|\nabla f| \leq \epsilon$,

$$
\max _{y \in \partial D_{R}}|\operatorname{Re}(f(y)-c)| \leq \epsilon / 2 \text { and } \max _{y \in \partial D_{R}}|\operatorname{Im}(f(y)-c)| \leq \epsilon / 2,
$$

so that $|f-c| \leq \epsilon$ on $\partial\left(D_{R} \backslash D_{\delta}\right)$. The maximum principle then gives (2.2).

Note that Lemma 2.1 does not hold for harmonic functions (in particular, (2.4)); e.g., take $\epsilon \log r /(4 \pi)$ and $R>\mathrm{e}^{8 \pi} \delta$. Clearly, Lemma 2.1 holds for 
the real part of a holomorphic function. However, on an annulus, not every harmonic function can be written this way.

It is not hard to see that $\left|f^{\prime}\left(z_{0}\right)\right| \leq \epsilon\left(1 / R+\delta\left|z_{0}\right|^{-2}\right) / \pi$ for $z_{0} \in D_{R / 2} \backslash$ $D_{2 \delta}$. It is not clear whether a corresponding estimate holds when there is a slit.

\section{Minimal annuli with slits.}

We will now bound the oscillation of the Gauss map of a double-valued minimal graph over an annulus with a slit. Double-valued on the slit. Fix $0<\alpha<1,0<\delta<R / 4,0<\epsilon$. Suppose $\Omega \subset\left\{x_{3}=0\right\}$ is a topological annulus,

$$
\begin{gathered}
\left|u_{+}(t)-u_{-}(t)\right|+t\left|\nabla u_{+}(t)-\nabla u_{-}(t)\right| \leq \epsilon \delta(t / \delta)^{\alpha} \text { for } \delta \leq t \leq \min \left\{R_{ \pm}\right\} \\
|A|(x) \leq \epsilon / r \text { for } x \in \Sigma
\end{gathered}
$$

where $r^{2}=x_{1}^{2}+x_{2}^{2}+x_{3}^{2}$. In Lemma 3.9 , we will see that $\Omega$ is given in polar coordinates by

$$
\left\{(\rho, \theta) \mid \rho_{\delta}(\theta) \leq \rho \leq \rho_{R}(\theta) \text { and } 0 \leq \theta \leq 2 \pi\right\},
$$

where $\rho_{\delta}$ and $\rho_{R}$ are functions of $\theta$ and, by convention, $\theta=0$ and $\theta=2 \pi$ correspond to $u_{-}$and $u_{+}$, respectively. Hence, $u$ is double-valued over the $x_{1}$-axis from $\delta$ to $\min \left\{R_{ \pm}\right\}$(the "slit") with values $u_{ \pm}$and $\nabla u_{ \pm}$for $u$ and $\nabla u=\left(\partial_{1} u, \partial_{2} u\right)$, respectively, and $\partial \Omega$ has corners (e.g., if $\left.\rho_{\delta}(0) \neq \rho_{\delta}(2 \pi)\right)$. Let $\Pi: \mathbf{S}^{2} \backslash\{(0,0,-1)\} \rightarrow \mathbf{C}$ be the stereographic projection so $\Pi(x)=$ $\left(x_{1}+i x_{2}\right) /\left(1+x_{3}\right)$ and if $a, a^{\prime} \in \mathcal{B}_{\pi / 4}(0,0,1)$, then

$$
\left|\Pi(a)-\Pi\left(a^{\prime}\right)\right| \leq\left|a-a^{\prime}\right| \leq 2\left|\Pi(a)-\Pi\left(a^{\prime}\right)\right| .
$$

(See, e.g., (28) on page 20 of $[\mathrm{A}]$ for (3.8)).

Our arguments will be modelled on those for holomorphic functions in Section 2. New error terms will arise both from the presence of the slit and from the fact that $\Sigma$ is not flat. Set $\gamma_{t}=\partial B_{t} \cap \Sigma$ and let $\sigma_{t} \subset\left\{x_{3}=0\right\}$ be the orthogonal projection of $\gamma_{t}$. 
Lemma 3.9. If $\epsilon<1 /\left(54 \pi^{2}\right), \delta \leq t \leq R$, and $\Sigma$ satisfies (3.1)-(3.6), then each $\sigma_{t}$ is a radial graph of a function $\rho_{t}$ of $\theta$ with $5 t / 6 \leq \rho_{t} \leq t$, and

$$
\begin{aligned}
u^{2}\left(x_{1}, x_{2}\right) & \leq\left(x_{1}^{2}+x_{2}^{2}\right) / 36 \text { for }\left(x_{1}, x_{2}\right) \in \Omega, \\
|\nabla u| & \leq 3 \pi \epsilon \text { and }|u| \leq 5 \pi^{2} \epsilon \delta \text { on } \bar{D}_{\delta} \cap \Omega, \\
\left|\rho_{t}(2 \pi)-\rho_{t}(0)\right| & <\epsilon \delta(t / \delta)^{\alpha} .
\end{aligned}
$$

Proof. By (3.1) and (3.6), we have

$$
|\nabla| \nabla u||\left(x_{1}, x_{2}\right) \leq|A|\left(x_{1}, x_{2}, u\right)\left(1+|\nabla u|^{2}\right)^{3 / 2}<9 \epsilon\left(x_{1}^{2}+x_{2}^{2}\right)^{-1 / 2} / 8 .
$$

Combining (3.3) and (3.13),

$$
\begin{aligned}
& \max _{\partial D_{\delta}}|\nabla u| \leq\left|\nabla u_{-}(\delta)\right|+2 \pi \delta \max _{\partial D_{\delta}}|\nabla| \nabla u|| \leq 9 \pi \epsilon / 4, \\
& \max _{\partial D_{\delta}}|u| \leq\left|u_{-}(\delta)\right|+2 \pi \delta \max _{\partial D_{\delta}}|\nabla u| \leq 9 \pi^{2} \epsilon \delta / 2<\delta / 12 .
\end{aligned}
$$

Integrating $|\nabla u|<1 / 6$ along rays, (3.15) implies that

$$
u^{2}\left(x_{1}, x_{2}\right) \leq\left(x_{1}^{2}+x_{2}^{2}\right) / 36 \text { for }\left(x_{1}, x_{2}\right) \in \Omega \cap\left\{\left(x_{1}^{2}+x_{2}^{2}\right)^{1 / 2}>5 \delta / 6\right\} .
$$

Since $25 \delta^{2}(1+1 / 36) / 36<\delta^{2}$ and $\Omega$ is connected, it follows that $\Omega \subset$ $\left\{\left(x_{1}^{2}+x_{2}^{2}\right)^{1 / 2}>5 \delta / 6\right\}$ and (3.10) holds for all $\left(x_{1}, x_{2}\right) \in \Omega$. Define the function $r_{u}: \Omega \rightarrow \mathbf{R}$ by $r_{u}\left(x_{1}, x_{2}\right)=\left|\left(x_{1}, x_{2}, u\left(x_{1}, x_{2}\right)\right)\right|$. Using $|\nabla u|<1 / 6$ and (3.10), for any $\theta$,

$$
\partial r_{u}^{2} / \partial \rho=\partial / \partial \rho\left[\rho^{2}+u^{2}(\rho \cos \theta, \rho \sin \theta)\right]>r_{u}>\rho>0 .
$$

In particular, $\sigma_{t}$ is a radial graph $\rho_{t}(\theta)(\cos \theta, \sin \theta)$ with $5 t / 6 \leq \rho_{t}(\theta) \leq t$. Integrating (3.13) along rays from $\partial D_{\delta}$ and using (3.14)-(3.15) gives (3.11). Using (3.5), the definition of $\rho_{t}$, and $\left|u_{ \pm}\right|(t) \leq t / 6$,

$$
\begin{aligned}
& \left|\left(\rho_{t}^{2}(2 \pi)+u_{-}^{2}\left(\rho_{t}(2 \pi)\right)\right)^{1 / 2}-t\right| \\
& \leq\left|\rho_{t}^{2}(2 \pi)+u_{-}^{2}\left(\rho_{t}(2 \pi)\right)-t^{2}\right| / t \\
& =\left|u_{-}^{2}\left(\rho_{t}(2 \pi)\right)-u_{+}^{2}\left(\rho_{t}(2 \pi)\right)\right| / t \\
& \leq\left|u_{-}\left(\rho_{t}(2 \pi)\right)-u_{+}\left(\rho_{t}(2 \pi)\right)\right| / 3 \leq \epsilon \delta(t / \delta)^{\alpha} / 3 .
\end{aligned}
$$

Finally, since $\frac{d}{d t}\left(t^{2}+u_{-}^{2}(t)\right)^{1 / 2}>1 / 2($ by $(3.17)),(3.18)$ implies that

$$
\left|\rho_{t}(2 \pi)-\rho_{t}(0)\right|<2\left|\left(\rho_{t}^{2}(2 \pi)+u_{-}^{2}\left(\rho_{t}(2 \pi)\right)\right)^{1 / 2}-t\right|<\epsilon \delta(t / \delta)^{\alpha} .
$$


Lemma 3.20. If $\epsilon<1 /\left(54 \pi^{2}\right), \delta \leq t \leq R$, and $\Sigma$ satisfies (3.1)-(3.6), then

$$
\begin{gathered}
2 \pi-C_{0} \epsilon \leq \delta^{-1} \int_{\gamma_{\delta}}\left|\nabla_{\Sigma} r\right|, \\
t^{-1} \operatorname{Length}\left(\gamma_{t}\right) \leq \min \left\{2 \pi+C_{0} \epsilon, 3 \pi\right\} .
\end{gathered}
$$

Proof. By Lemma 3.9, each $\sigma_{t}$ is a radial graph of $\rho_{t}$. Hence,

$$
\left(\rho_{t}(\theta)\right)^{2}+u^{2}\left(\rho_{t}(\theta) \cos \theta, \rho_{t}(\theta) \sin \theta\right)=t^{2} .
$$

Differentiating (3.23) (with respect to $\theta$ ), we get

$$
\rho_{t} \partial_{\theta} \rho_{t}+u\langle\nabla u,(\cos \theta, \sin \theta)\rangle \partial_{\theta} \rho_{t}=-u \rho_{t}\langle\nabla u,(-\sin \theta, \cos \theta)\rangle .
$$

Substituting $5 t / 6 \leq \rho_{t} \leq t,|\nabla u|<1 / 6$, and $|u| \leq t / 6$ on $\sigma_{t}$,

$$
\left|\partial_{\theta} \rho_{t}\right| \leq|u| t|\nabla u| /\left[\rho_{t}-|u||\nabla u|\right] \leq 3|u||\nabla u| / 2 \leq t / 24 .
$$

By (3.25) (and since $\left.\rho_{t} \leq t\right),\left|d \sigma_{t} / d \theta\right|=\left|(\cos \theta, \sin \theta) \partial_{\theta} \rho_{t}+\rho_{t}(-\sin \theta, \cos \theta)\right|$ $<25 t / 24$. Combining this with $|\nabla u|<1 / 6$ implies that

$$
\operatorname{Length}\left(\gamma_{t}\right) \leq \int_{0}^{2 \pi}\left|\frac{d \sigma_{t}}{d \theta}\right|\left(1+|\nabla u|^{2}\right)^{1 / 2} d \theta<5 \pi t / 2 .
$$

The triangle inequality and (3.11) imply that $\rho_{\delta} \geq \delta-\max _{\sigma_{\delta}}|u| \geq(1-$ $\left.5 \pi^{2} \epsilon\right) \delta$, so

$$
\operatorname{Length}\left(\gamma_{\delta}\right) \geq \operatorname{Length}\left(\sigma_{\delta}\right) \geq 2 \pi \min \rho_{\delta} \geq\left(2 \pi-C_{1} \epsilon\right) \delta .
$$

Given $x=\left(x_{1}, x_{2}, x_{3}\right) \in \gamma_{\delta}$, then $\left(x_{1}, x_{2}\right) \in \sigma_{\delta} \subset \bar{D}_{\delta}$ and (3.11) give

$$
|\langle\mathbf{n}(x), x / \delta\rangle|=\delta^{-1}\left(1+|\nabla u|^{2}\right)^{-1 / 2}\left|u-x_{1} \partial_{1} u-x_{2} \partial_{2} u\right|<C_{2} \epsilon .
$$

Consequently,

$$
\left|\nabla_{\Sigma} r\right|>1-C_{2} \epsilon \text { on } \gamma_{\delta} .
$$

Combining (3.27) and (3.29) gives (3.21) for any $C_{0} \geq C_{1}+2 \pi C_{2}$.

Below, we will need that the endpoints of $\gamma_{t}$ are close. By (3.12), $\left|\rho_{t}(2 \pi)-\rho_{t}(0)\right|<\epsilon \delta(t / \delta)^{\alpha}<\epsilon t$. Therefore, by the triangle inequality, $|\nabla u|<1 / 6$, and (3.5), we get

$$
\begin{aligned}
& \left|u_{+}\left(\rho_{t}(2 \pi)\right)-u_{-}\left(\rho_{t}(0)\right)\right| \\
& \leq\left|u_{+}\left(\rho_{t}(2 \pi)\right)-u_{+}\left(\rho_{t}(0)\right)\right|+\left|u_{+}\left(\rho_{t}(0)\right)-u_{-}\left(\rho_{t}(0)\right)\right| \\
& \leq \epsilon t / 6+\epsilon t=7 \epsilon t / 6 .
\end{aligned}
$$


We conclude that the distance between the endpoints of $\gamma_{t}$ is at most $2 \epsilon t$.

Let $P_{t}$ be the plane orthogonal to $\mathbf{n}\left(\gamma_{t} \cap \gamma_{-}\right)$through $\gamma_{t} \cap \gamma_{-}$. Let $\hat{\sigma}_{t}$ be the (orthogonal) projection of $\gamma_{t}$ to $P_{t}$. Since $|\nabla u|<1 / 6, \Sigma$ is a multivalued graph over (a subset of) $P_{t}$ of a function $w$. To complete the proof, we will argue as above to bound the length of $\hat{\sigma}_{t}$ and $|\nabla w|$ on $\hat{\sigma}_{t}$. Using (3.26), Length $\left(\hat{\sigma}_{t}\right)<5 \pi t / 2$. Hence, as in (3.13)-(3.15), we get

$$
\begin{aligned}
\max _{\hat{\sigma}_{t}}|\nabla w| & \leq C_{3} \epsilon, \\
\max _{\hat{\sigma}_{t}}|w| & \leq 5 \pi C_{3} \epsilon t / 2 .
\end{aligned}
$$

It remains to bound the length of $\hat{\sigma}_{t}$ by $2 \pi t$ plus a multiple of $\epsilon t$. Using $|\nabla u|<1 / 6$ and (3.10), $B_{t} \cap P_{t} \subset\left\{\left|x_{3}\right|<t / 2\right\}$. In particular, $\partial B_{t}$ intersects $P_{t}$ with slope at least $\sqrt{3}$ (in absolute value) over $\left\{x_{3}=0\right\}$. Since the slope of $P_{t}$ is less than $1 / 6$ over $\left\{x_{3}=0\right\}$, this implies that $\left|\nabla_{P_{t}} r\right| \geq C_{4}>0$ on $\partial B_{t} \cap P_{t}$. Choose polar coordinates $\hat{\rho}, \hat{\theta}$ on $P_{t}$ so $\partial B_{t} \cap P_{t}$ is a circle $\hat{\rho}=$ Const $\leq t$. Arguing as in (3.17), we get, for any $\hat{\theta}$,

$$
\partial / \partial \hat{\rho}\left[\hat{\rho}^{2}+w^{2}(\hat{\rho} \cos \hat{\theta}, \hat{\rho} \sin \hat{\theta})\right]>C_{5} \hat{\rho}>0 .
$$

Hence, $\hat{\sigma}_{t}$, like $\sigma_{t}$, is a connected (multi-valued) radial graph of a function $\hat{\rho}_{t}$ of $\hat{\theta}$ satisfying (3.23)-(3.25). From (3.25), (3.31), and (3.32), we get that $\left|\partial_{\hat{\theta}} \hat{\rho}_{t}\right| \leq C_{6} \epsilon t$. Inserting this and (3.31) into (3.26) (with $w$ in place of $u$ ) gives (3.22) since the domain of $\hat{\rho}_{t}$ is contained in $\{\hat{\theta} \mid-C \epsilon \leq \hat{\theta} \leq 2 \pi+C \epsilon\}$. This followed since the distance between the endpoints of $\gamma_{t}$ is at most $2 \epsilon t$ (and orthogonal projection is distance nonincreasing).

The key point in the next theorem, our main result, is that the constants are independent of $\delta$ and $R$. The proof follows Lemma 2.1. If $\Sigma$ is as in (3.1), then the Gauss map $\mathbf{n}: \Sigma \rightarrow \mathcal{B}_{\pi / 4}(0,0,1) \subset \mathbf{S}^{2}$ is conformal. Composing $\mathbf{n}$ with the stereographic projection $\Pi$ and using (3.8), we get a conformal map

$$
f=\Pi(\mathbf{n}(\cdot)): \Sigma \rightarrow D_{1}(0) \subset \mathbf{C} \text { with }\left|\nabla_{\Sigma} f\right| \leq|A| .
$$

Note that, if $x, y \in \Omega$, then

$$
\begin{aligned}
|f(x, u(x))-f(y, u(y))| & =\left|\frac{\nabla u(x)}{1+\left(1+|\nabla u(x)|^{2}\right)^{1 / 2}}-\frac{\nabla u(y)}{1+\left(1+|\nabla u(y)|^{2}\right)^{1 / 2}}\right| \\
& \leq 2|\nabla u(x)-\nabla u(y)| .
\end{aligned}
$$


Theorem 3.36. There are $C(\alpha), \epsilon_{0}(\alpha)>0$ so that if $\epsilon<\epsilon_{0}$ and $\Sigma$ satisfies (3.1)-(3.6), then

$$
\max _{z \in \Sigma}|\mathbf{n}(z)-(0,0,1)| \leq C \epsilon
$$

Proof. We will prove (3.37) by bounding $|f|$. For $\delta \leq s \leq R$, set

$$
I(s)=s^{-1} \int_{\gamma_{s}} f\left|\nabla_{\Sigma} r\right|=\int_{\gamma_{s}} f \frac{d \log r}{d n} .
$$

We will show that $I(s)$ is almost constant. By Stokes' theorem and the co-area formula,

$$
\begin{aligned}
I(s)-I(\delta)= & \int_{B_{s} \cap \Sigma}\left\langle\nabla_{\Sigma} f, \nabla_{\Sigma} \log r\right\rangle+\int_{B_{s} \cap \Sigma} f \Delta_{\Sigma} \log r \\
& -\int_{B_{s} \cap\left(\gamma_{+} \cup \gamma_{-}\right)} f \frac{d \log r}{d n} \\
= & \int_{\delta}^{s} t^{-1} \int_{\gamma_{t}}\left|\nabla_{\Sigma} r\right|^{-1}\left\langle\nabla_{\Sigma} f, \nabla_{\Sigma} r\right\rangle d t \\
& +\int_{B_{s} \cap \Sigma} f \Delta_{\Sigma} \log r-\int_{\delta}^{s_{0}}\left[f_{+} F_{+}-f_{-} F_{-}\right](t) d t \\
& -\int_{\delta_{+}}^{\delta} f_{+}(t) F_{+}(t) d t-\int_{s_{0}}^{s_{+}} f_{+}(t) F_{+}(t) d t \\
& +\int_{s_{0}}^{s_{-}} f_{-}(t) F_{-}(t) d t .
\end{aligned}
$$

Here $s_{-}=\rho_{s}(0), s_{+}=\rho_{s}(2 \pi)$ (so that $\left.s^{2}=s_{ \pm}^{2}+u_{ \pm}^{2}\left(s_{ \pm}\right)\right), s_{0}=\min \left\{s_{+}, s_{-}\right\}$, and

$$
\begin{aligned}
f_{ \pm}(t)= & f\left(t, 0, u_{ \pm}(t)\right) \\
F_{ \pm}(t)= & \left\langle\frac{\left(-\partial_{1} u_{ \pm} \partial_{2} u_{ \pm}, 1+\left(\partial_{1} u_{ \pm}\right)^{2}, \partial_{2} u_{ \pm}\right)}{\left(\left|\partial_{1} u_{ \pm} \partial_{2} u_{ \pm}\right|^{2}+\left(1+\left|\partial_{1} u_{ \pm}\right|^{2}\right)^{2}+\left|\partial_{2} u_{ \pm}\right|^{2}\right)^{1 / 2}}, \frac{\left(t, 0, u_{ \pm}\right)}{t^{2}+u_{ \pm}^{2}}\right\rangle \\
& \cdot\left(1+\left|\partial_{1} u_{ \pm}\right|^{2}\right)^{1 / 2} \\
= & \frac{\partial_{2} u_{ \pm}}{\left(1+\left|\nabla u_{ \pm}\right|^{2}\right)^{1 / 2}}\left[\frac{-t \partial_{1} u_{ \pm}+u_{ \pm}}{t^{2}+u_{ \pm}^{2}}\right] .
\end{aligned}
$$

In (3.41), $\partial_{i} u_{ \pm}(t)$ denote the values of $\partial_{i} u(t, 0)$. When $\Sigma$ is an annulus in the plane, the the last five terms in (3.39) don't appear and the first term in 
(3.39) vanishes since $f$ is conformal (see (2.4)); we will bound these terms in general in Lemma 3.45 below.

Lemmas 3.9, 3.20 give bounds for $|\nabla u|$ along $\gamma_{\delta}$ and the length of $\gamma_{\delta}$; together this bounds $|I(\delta)|$. Combining this with the bound on $I(s)-I(\delta)$ from Lemma 3.45 , we get

$$
|I(s)| \leq|I(\delta)|+|I(s)-I(\delta)| \leq C_{3}^{\prime} \epsilon .
$$

By (3.47) and (3.42) (and since $\gamma_{s}$ is connected), there exist $y_{s} \in \gamma_{s}$ with

$$
\left|\operatorname{Re}\left(f\left(y_{s}\right)\right)\right| \leq\left|\int_{\gamma_{s}} f\right| \nabla_{\Sigma} r|| / \int_{\gamma_{s}}\left|\nabla_{\Sigma} r\right| \leq|I(s)| /\left(2 \pi-C_{5} \epsilon\right) \leq C_{4}^{\prime} \epsilon
$$

so long as $\epsilon<2 \pi / C_{5}$. Combining (3.43) with the gradient bound (3.34), the length bound on $\gamma_{s}$ from Lemma 3.20, and (3.6), we get

$$
\max _{y \in \gamma_{s}}|\operatorname{Re}(f(y))| \leq C_{4}^{\prime} \epsilon+3 \pi s \sup _{\gamma_{s}}|A| \leq C_{4} \epsilon .
$$

Repeating this, (3.44) holds also for $\max _{\gamma_{s}}|\operatorname{Im}(f)|$ so $|f| \leq \sqrt{2} C_{4} \epsilon$ on $\Sigma$, giving (3.37).

We will now show that $I(s)$ defined in (3.38) is almost constant.

Lemma 3.45. With the notation as in Theorem 3.36 and its proof (see (3.34) and (3.38))

$$
\begin{aligned}
|I(s)-I(\delta)| & \leq C_{3} \epsilon \\
2 \pi-C_{5} \epsilon & \leq s^{-1} \int_{\gamma_{s}}\left|\nabla_{\Sigma} r\right| .
\end{aligned}
$$

Proof. To get (3.46), it suffices to bound the six terms in (3.39). We begin with the last three. Since $|\nabla u|<1 / 6$ and $\left|u_{ \pm}(t)\right| \leq t / 6$ (by (3.10)), we get $\left|F_{ \pm}(t)\right| \leq\left|\nabla u_{ \pm}\right| /(3 t)<1 /(18 t)$. By this, $|f|<1$, and $\left|\nabla u_{+}(t)\right| \leq 3 \pi \epsilon$ for $t \leq \delta$ (by $(3.11))$, we get

$$
\int_{\delta_{+}}^{\delta}\left|f_{+}(t) F_{+}(t)\right| d t \leq \int_{\delta_{+}}^{\delta}\left|F_{+}(t)\right| d t \leq \int_{\delta_{+}}^{\delta} \frac{\left|\nabla u_{+}\right|}{3 t} d t<\epsilon,
$$

where we also used that $\delta_{+}>5 \delta / 6$. By $(3.12),\left|\rho_{s}(2 \pi)-\rho_{s}(0)\right|<\epsilon \delta(s / \delta)^{\alpha}$. Therefore, since $|f|<1$ and $\left|F_{ \pm}(t)\right| \leq\left|\nabla u_{ \pm}\right| /(3 t)<1 /(18 t)$,

$$
\int_{s_{0}}^{s_{ \pm}}\left|f_{ \pm} F_{ \pm}\right|(t) d t \leq \int_{s_{0}}^{s_{ \pm}}\left|F_{ \pm}(t)\right| d t<\frac{\epsilon \delta}{18 s_{0}}\left(\frac{s}{\delta}\right)^{\alpha}<\epsilon\left(\frac{\delta}{s}\right)^{1-\alpha} \leq \epsilon
$$


Let $\gamma_{t}^{\prime}$ be the unit tangent to $\gamma_{t}$. For the first term in (3.39), since $f$ is conformal and $\nabla_{\Sigma} r /\left|\nabla_{\Sigma} r\right|$ is the unit normal to $\gamma_{t}$,

$$
\int_{\gamma_{t}}\left|\nabla_{\Sigma} r\right|^{-1}\left\langle\nabla_{\Sigma} f, \nabla_{\Sigma} r\right\rangle=i \int_{\gamma_{t}}\left\langle\nabla_{\Sigma} f, \gamma_{t}^{\prime}\right\rangle=i\left[f_{+}\left(t_{+}\right)-f_{-}\left(t_{-}\right)\right],
$$

where $t^{2}=t_{ \pm}^{2}+u_{ \pm}^{2}\left(t_{ \pm}\right)$as before. By (3.5), (3.6), (3.34), (3.35), and (3.12),

$$
\begin{aligned}
\left|f_{+}\left(t_{+}\right)-f_{-}\left(t_{-}\right)\right| & \leq\left|f_{+}\left(t_{+}\right)-f_{-}\left(t_{+}\right)\right|+\left|f_{-}\left(t_{+}\right)-f_{-}\left(t_{-}\right)\right| \\
& \leq 2 \epsilon\left(\delta / t_{+}\right)^{1-\alpha}+\epsilon\left|t_{+}-t_{-}\right| / t_{0}<4 \epsilon(\delta / t)^{1-\alpha}
\end{aligned}
$$

where $t_{0}=\min \left\{t_{+}, t_{-}\right\}>5 t / 6$. Hence, (3.50) and (3.51) give

$$
\left.\left|\int_{\delta}^{s} t^{-1} \int_{\gamma_{t}}\right| \nabla_{\Sigma} r\right|^{-1}\left\langle\nabla_{\Sigma} f, \nabla_{\Sigma} r\right\rangle d t \mid \leq 4 \int_{\delta}^{s} t^{-1} \epsilon(\delta / t)^{1-\alpha} d t \leq \frac{4 \epsilon}{1-\alpha} .
$$

Note that, since $\Sigma$ is minimal,

$$
\Delta_{\Sigma} \log r=2\left(1-\left|\nabla_{\Sigma} r\right|^{2}\right) r^{-2} \geq 0 .
$$

Using $|f|<1$, (3.53), and Stokes' theorem, the second term in (3.39) is bounded by

$$
\begin{aligned}
\left|\int_{B_{s} \cap \Sigma} f \Delta_{\Sigma} \log r\right| \leq & \int_{B_{s} \cap \Sigma} \Delta_{\Sigma} \log r \leq s^{-1} \int_{\gamma_{s}}\left|\nabla_{\Sigma} r\right| \\
& -\delta^{-1} \int_{\gamma_{\delta}}\left|\nabla_{\Sigma} r\right|+\left|\int_{B_{s} \cap\left(\gamma_{+} \cup \gamma_{-}\right)} \frac{d \log r}{d n}\right| \\
\leq & s^{-1} \int_{\gamma_{s}}\left|\nabla_{\Sigma} r\right|-\delta^{-1} \int_{\gamma_{\delta}}\left|\nabla_{\Sigma} r\right| \\
& +\int_{\delta}^{s_{0}}\left|F_{+}(t)-F_{-}(t)\right| d t+\int_{\delta_{+}}^{\delta}\left|F_{+}(t)\right| d t \\
& +\int_{s_{0}}^{s_{+}}\left|F_{+}(t)\right| d t+\int_{s_{0}}^{s_{-}}\left|F_{-}(t)\right| d t .
\end{aligned}
$$

We next bound $\left|F_{+}(t)-F_{-}(t)\right|$. Given $p, t \in \mathbf{R}$ with $|p| \leq t / 6$ and $q \in$ $D_{1 / 6}(0) \subset \mathbf{R}^{2}$, define $F_{1}(q)=q_{2}\left(1+q_{1}^{2}+q_{2}^{2}\right)^{-1 / 2}$, and $F_{2}(t, p, q)=(p-$ $\left.q_{1} t\right)\left(t^{2}+p^{2}\right)^{-1}$, so that

$$
F_{ \pm}(t)=F_{1}\left(\nabla u_{ \pm}\right) F_{2}\left(t, u_{ \pm}, \nabla u_{ \pm}\right)
$$


Keeping in mind that $|p| \leq t / 6$ and $|q|<1 / 6$, it is easy to see that

$$
\begin{aligned}
\left|\nabla F_{1}\right|^{2} & =\left(q_{1}^{2} q_{2}^{2}+\left(1+q_{1}^{2}\right)^{2}\right) /\left(1+|q|^{2}\right)^{3} \\
& \leq\left(1+q_{1}^{2}\right)\left(1+|q|^{2}\right) /\left(1+|q|^{2}\right)^{3} \leq 1, \\
\left|\partial_{p} F_{2}\right| & =\left[t^{2}-p^{2}+2 p q_{1} t\right] /\left(t^{2}+p^{2}\right)^{2} \\
& \leq\left(t^{2}+q_{1}^{2} t^{2}\right) /\left(t^{2}+p^{2}\right)^{2} \leq 2 / t^{2}, \\
\left|\partial_{q_{1}} F_{2}\right| & =t /\left(t^{2}+p^{2}\right) \leq 1 / t, \text { and } \partial_{q_{2}} F_{2} \equiv 0 .
\end{aligned}
$$

Since $\left|F_{1}\right|<1 / 6$ and $\left|F_{2}\right| \leq 1 /(3 t),(3.5),(3.55)$, and (3.56) imply that

$$
\begin{aligned}
\left|F_{+}(t)-F_{-}(t)\right| \leq & \left|F_{1}\left(\nabla u_{+}\right) F_{2}\left(t, u_{+}, \nabla u_{+}\right)-F_{1}\left(\nabla u_{-}\right) F_{2}\left(t, u_{+}, \nabla u_{+}\right)\right| \\
& +\left|F_{1}\left(\nabla u_{-}\right) F_{2}\left(t, u_{+}, \nabla u_{+}\right)-F_{1}\left(\nabla u_{-}\right) F_{2}\left(t, u_{+}, \nabla u_{-}\right)\right| \\
& +\left|F_{1}\left(\nabla u_{-}\right) F_{2}\left(t, u_{+}, \nabla u_{-}\right)-F_{1}\left(\nabla u_{-}\right) F_{2}\left(t, u_{-}, \nabla u_{-}\right)\right| \\
\leq & \left|\nabla u_{+}(t)-\nabla u_{-}(t)\right|\left(\max \left|\nabla F_{1}\right| /(3 t)+\max \left|\nabla_{q} F_{2}\right| / 6\right) \\
& +\left|u_{+}(t)-u_{-}(t)\right| \max \left|\partial_{p} F_{2}\right| / 6 \\
\leq & \epsilon \delta^{1-\alpha} t^{\alpha-2} .
\end{aligned}
$$

Combining Lemma 3.20, (3.48), (3.49), and (3.57), (3.54) becomes

$$
\left|\int_{B_{s} \cap \Sigma} f \Delta_{\Sigma} \log r\right| \leq 2 C_{0} \epsilon+3 \epsilon+\epsilon \delta^{1-\alpha} \int_{\delta}^{s_{0}} t^{\alpha-2} d t \leq C_{5} \epsilon .
$$

As in (3.58), Lemma 3.20, Stokes' theorem, (3.53), (3.48), (3.49), and (3.57) imply that

$$
s^{-1} \int_{\gamma_{s}}\left|\nabla_{\Sigma} r\right| \geq \delta^{-1} \int_{\gamma_{\delta}}\left|\nabla_{\Sigma} r\right|-\left|\int_{B_{s} \cap\left(\gamma_{+} \cup \gamma_{-}\right)} \frac{d \log r}{d n}\right| \geq 2 \pi-C_{5} \epsilon
$$

giving (3.47).

Combining $|f|<1,\left|F_{ \pm}(t)\right| \leq 1 /(18 t)$, (3.5), (3.35), and (3.57), we have

$$
\begin{aligned}
\left|f_{+} F_{+}-f_{-} F_{-}\right|(t) & \leq\left|f_{+} F_{+}-f_{+} F_{-}\right|(t)+\left|f_{+} F_{-}-f_{-} F_{-}\right|(t) \\
& <\epsilon \delta^{1-\alpha} t^{\alpha-2}+1 /(9 t) \epsilon \delta^{1-\alpha} t^{\alpha-1}
\end{aligned}
$$

For the remaining term in (3.39), we integrate (3.60) to get

$$
\int_{\delta}^{s_{0}}\left|f_{+} F_{+}-f_{-} F_{-}\right|(t) d t<2 \epsilon \delta^{1-\alpha} \int_{\delta}^{s_{0}} t^{\alpha-2} d t \leq 2 \epsilon /(1-\alpha) .
$$

Finally, substituting (3.48), (3.49), (3.52), (3.58), and (3.61) into (3.39), we get (3.46). 


\section{Appendix A. A fact about qausi-conformal maps.}

The following lemma is a special case of the fact that quasi-conformal maps are open (see, e.g., [V]); we include for completeness a proof of this lemma.

Lemma A.1. If $\Sigma$ is compact, connected, $|A|^{2} \leq C|K|, \Omega \subset \mathbf{S}^{2}$ is a connected open set with $\partial \Omega$ piecewise smooth and compact, $\bar{\Omega} \cap \mathbf{n}(\partial \Sigma)=\emptyset$, and $\mathcal{H}^{1}(\Omega \cap \mathbf{n}(\Sigma)) \neq 0$, then $\Omega \subset \mathbf{n}(\Sigma)$.

Proof. Set $J=\{y \in \Sigma \mid K(y)=0\} . \mathbf{n}(J)$ is closed and $\Omega \cap \mathbf{n}(\Sigma) \backslash \mathbf{n}(J)$ is (relatively) closed in $\Omega \backslash \mathbf{n}(J)$. Since $|A|^{2} \leq C|K|$, the differential of $\mathbf{n}$ vanishes on $J$. By the general Morse-Sard-Federer theorem, $\mathcal{H}^{1}(\mathbf{n}(J))=$ 0 (see $[\mathrm{F}]$ theorem 3.4.3). Hence, $\Omega \backslash \mathbf{n}(J)$ is connected (and open) and $\Omega \cap \mathbf{n}(\Sigma) \backslash \mathbf{n}(J) \neq \emptyset$. Since the implicit function theorem implies that $\Omega \cap \mathbf{n}(\Sigma) \backslash \mathbf{n}(J)$ is also open, $\Omega \backslash \mathbf{n}(J) \subset \mathbf{n}(\Sigma)$, giving the claim.

\section{References.}

[A] L.V. Ahlfors, Complex analysis. An introduction to the theory of analytic functions of one complex variable, Third edition, International Series in Pure and Applied Mathematics, McGraw-Hill Book Co., New York, 1978.

[CM] T.H. Colding and W.P. Minicozzi II, Minimal surfaces, Courant Lecture Notes in Math., 4 (1999).

[F] H. Federer, Geometric measure theory, Die Grundlehren der mathematischen Wissenschaften, Band 153, Springer-Verlag New York Inc., New York, 1969.

[V] J. Väisälä, A survey of quasiregular maps in $\mathbf{R}^{n}$, Proceedings of the International Congress of Mathematicians (Helsinki, 1978), 685-691, Acad. Sci. Fennica, Helsinki, 1980. 
Courant Institute of Mathematical Sciences

251 Mercer Street, New York, NY 10012

and

MIT

77 Massachusetts Avenue

CAMBridge, MA 02139-4307

E-mail address: colding@cims.nyu.edu

Department of Mathematics

JOHNS HOPKINS UNIVERSITY

3400 N. Charles St.

BALTIMORE, MD 21218

E-mail address: minicozz@math.jhu.edu 
